\title{
Can sutures get wet? Prospective randomised controlled trial of wound management in general practice
}

Clare Heal, Petra Buettner, Beverly Raasch, Sheldon Browning, David Graham, Rachel Bidgood, Margaret Campbell, Robert Cruikshank

\begin{abstract}
Objective To compare standard management of keeping wounds dry and covered with allowing wounds to be uncovered and wet in the first 48 hours after minor skin excision.

Design Prospective, randomised controlled, multicentre trial testing for equivalence of infection rates.

Setting Primary care in regional centre, Queensland, Australia. Participants 857 patients randomised to either keep their wound dry and covered $(\mathrm{n}=442)$ or remove the dressing and wet the wound $(\mathrm{n}=415)$.

Results The incidence of infection in the intervention group $(8.4 \%)$ was not inferior to the incidence in the control group $(8.9 \%)(\mathrm{P}<0.05)$. The one sided 95\% confidence interval for the difference of infection rates was $\infty$ to 0.028 .

Conclusion These results indicate that wounds can be uncovered and allowed to get wet in the first 48 hours after minor skin excision without increasing the incidence of infection.
\end{abstract}

\section{Introduction}

Guidelines for managing surgical wounds that are closed primarily (that is, those with the skin edges re-approximated at the end of the procedure) instruct that patients should keep their wounds dry and covered for 24-48 hours. ${ }^{1}$ Before our study, the four participating general practices were implementing these guidelines and advising patients to keep their wounds dry and covered for 48 hours after minor excisions. For patients living in the tropics of North Queensland, with increased heat and humidity, this recommendation is impractical and a nuisance.

Literature on wound management is sparse. As regards wetting sutures, previous studies have compared standard management (keeping wounds dry) with washing with soap and water in the first 48 hours after minor skin excisions or compared standard management with early showering after more major surgery. $^{2-5}$ These relatively few published studies suggest that getting sutures wet does not increase the infection rate. However, numbers of patients studied have been small, and only one previous study was randomised. No previous studies have been done in the general practice setting.

As regards uncovering sutures, some evidence shows that no difference exists in the incidence of infection between wounds left without dressings and those covered with a dry dressing in the early postoperative period. ${ }^{6-8}$ Again, no previous studies had been done in a general practice setting.

As the two factors, wetting and uncovering, are difficult to separate in the immediate postoperative period, we decided to assess these factors together. We proposed to look at the effects of allowing patients to uncover and wet their wounds during the first 48 hours after minor skin excision, hypothesising that infection rates would be non-inferior compared with a control group following the dry wound management recommendations.

\section{Methods}

\section{Study design}

This was a randomised controlled, multicentre trial involving patients presenting for minor skin excisions.

\section{Setting and participants}

Sixteen general practitioners from four practices in the Mackay area, tropical North Queensland, Australia (latitude 21 $1^{\circ}$ south; inhabitants of Mackay area approximately 75000$)$ participated. The general practitioners were a self selected group who attended a monthly evidence based medicine meeting. Data collection took place from October 2004 to May 2005. We invited consecutive patients presenting for minor skin excisions to take part in the trial. Practice nurses were responsible for recruiting patients and collecting data. We collected demographic information on all patients, as well as clinical information on the presence or absence of diabetes or any other important medical condition (such as peripheral vascular disease, anaemia, or chronic obstructive pulmonary disease). We used a body site map to define excision sites. At the end of the study we asked practice nurses to re-examine computer records to fill in any missing data. The principal researcher visited participating general practitioners and practice nurses to provide training and ensure that recording was standardised.

We gave all participating patients an information sheet and asked them to give signed consent. We gave written instructions on postoperative wound care to patients who consented to participate.

\section{Eligibility criteria}

All patients who presented to a participating general practitioner for "minor skin excision," except for skin excisions on the face, were eligible to participate in the study. We excluded patients who were already taking oral antibiotics, for whom oral or topical antibiotics were clinically indicated immediately postoperatively, or who were on immunosuppressive drugs. Further exclusion criteria were lacerations, having a flap or two layer procedure (tying a "bleeder" did not count as two layer), excision of a sebaceous cyst, and skin excision on the face. We made these exclusions in an attempt to standardise the type of wound being studied and reduce the number of confounding factors. 


\section{Surgical wound management protocol}

A workshop attended by participating general practitioners developed guidelines to ensure that excisions were managed in a standardised manner. The following procedure was agreed: skin preparation (normal saline); usual sterile technique (standard precautions), including sterile gloves; local anaesthetic (type and volume recorded); suture material-nylon (size recorded); no antibiotics, either topical or oral (if required, or already prescribed, exclude from study), topical antiseptics (such as betadine or alcohol), or antiseptic washes or medicates soaps; dressing type--melolin and tape; removal of sutures, according to site (back $=10$ days, all other sites $=$ seven days).

\section{Intervention}

We gave patients oral and written instructions on postoperative wound management (fig 1). We asked the "dry" group to leave the dressing on and keep it dry for the first 48 hours, then to bathe and undress as normal until the sutures were taken out. We asked them to avoid using antiseptic washes or soaps.

We asked the wet group to take the dressing off within the first 12 hours and then bathe as normal until the sutures were taken out. We felt that patients should leave the surgery with a dressing to absorb immediate bleeding, but we also felt that defining an exact time to remove the dressing would be unrealistic; we considered "within 12 hours" to be a reasonable request. We also asked them to avoid using antiseptic washes and soaps.

\section{Clinical outcomes}

A practice nurse or doctor assessed wounds for infection on the day of removal of sutures, or sooner if the patient re-presented with a perceived infection. We adapted our definition of wound infection from standardised surveillance criteria for defining surgical site infections developed by the Centre for Disease Control's national nosocomial infection surveillance system (box). ${ }^{1}$ The primary researcher briefed all participating doctors and nurses on the definition of infection, and we also gave them written information.

\section{Sample size}

We calculated sample size on the basis of a pilot study done in February to June 2004 and involving 543 patients, which showed an overall infection rate of $5.7 \%$. On the basis of a projected infection rate of $5 \%$, we decided that an increase in incidence of infection of $5 \%$ would be clinically significant. To come to this conclusion with statistical confidence, a power of $80 \%$, and a significance level of 0.05 using a one sided equivalence test of proportions, we needed a total of 357 patients in the intervention group and 357 patients in the control group.

\section{Randomisation}

All patients provided written informed consent before enrolling in the study. After agreeing to participate, patients were randomised by picking a ball out of a hat. The practice nurses chose this method of randomisation because of its ease and acceptability. The practice nurses enrolled patients and assigned participants to their groups. No blinding took place.

\section{Statistical analysis}

We based all analyses on the intention to treat principle. We used $\chi^{2}$ tests to assess differences between categorical variables and unpaired $t$ tests to compare numerical and categorical variables. To determine non-inferiority, we calculated the one sided 95\% confidence interval of the difference in infection rates and compared it with the maximum allowable difference of $5 \%$. We considered $\mathrm{P}$ values less than 0.05 to be statistically significant.

\section{Results}

\section{Practice and study characteristics}

Participating general practitioners were younger (median age 44) and more predominantly female (64\%) than average for Australian general practitioners (median age category 45-54; $32 \%$ female). Of the total of 1247 patients who attended for skin excisions during the collection period from October 2004 to May 2005, we excluded 377 patients (table 1).

No significant differences existed in the age $(\mathrm{P}=0.87)$ or sex $(\mathrm{P}=0.89)$ of participating and non-participating patients. Of the remaining 870 patients, 450 patients were randomised to the intervention (wet) group and 420 to the control (dry) group. A total of 13 patients were eventually lost to follow-up. Follow-up was completed in 857 (98.5\%) randomised patients (fig 2).

\section{Baseline data}

We found no significant differences between the intervention group and the control group at baseline (table 2).

\section{Infections}

Infection occurred in $74(8.6 \%)$ of the 857 excisions. The intervention group had an infection rate of $8.4 \%$ compared with $8.9 \%$ in the control group. The one sided $95 \%$ confidence interval of the difference of the two proportions was $\infty$ to 0.028 , so the non-inferiority side was lower than 0.05 , the maximum allowable difference. We therefore concluded that the intervention group was not inferior to the control group with respect to the resulting infection rates $(\mathrm{P}<0.05)$.

\section{Discussion}

Our results indicate that patients can uncover and occasionally wet stitches in the first 48 hours after minor skin excisions without increasing the incidence of wound infection. The overall incidence of infection in our study was higher than we expected from our pilot study or from published literature looking at simi-

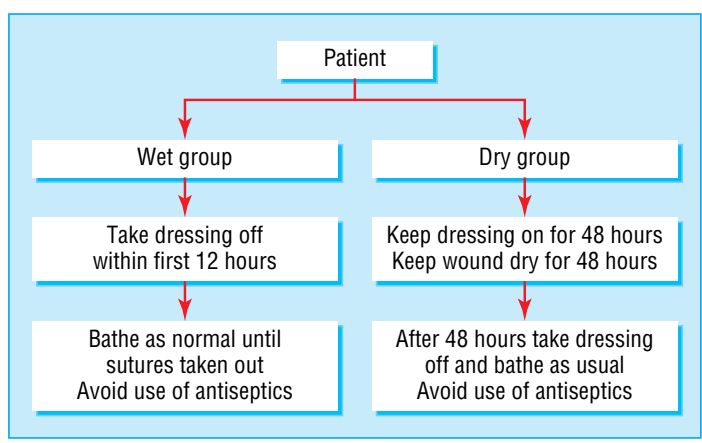

Fig 1 Wound management protocol in wet (intervention) and dry (control) groups 
Table 1 Reasons for exclusion of patients

\begin{tabular}{lc} 
Reason & No (\% of $\mathbf{1 2 4 7}$ eligible patients) \\
\hline Refused & $67(5.4)$ \\
\hline Forgot $^{\star}$ & $16(1.3)$ \\
\hline Not returning for removal of sutures ${ }^{* *}$ & $5(0.4)$ \\
\hline Face & $256(20.5)$ \\
\hline Biopsy & $10(0.8)$ \\
\hline Flap & $17(1.4)$ \\
\hline Immunosuppressed & $1(0.1)$ \\
\hline Antibiotics & $5(0.4)$ \\
\hline Total & $377(30.3)$
\end{tabular}

*The practice nurse forgot to invite these patients to participate.

**These patients knew that they would not be able to return for removal of sutures.

lar cohorts, ${ }^{910}$ although exclusion of facial excisions from our study, which may have a lower incidence of infection, may have falsely raised our overall incidence of infection.

The study did have several limitations. Various characteristics influence the occurrence of infections, and, although we recorded information on as many variables as possible, ensuring that baseline data were comparable proved difficult. For example, we had inadequate data recorded on suture size and occupation, so we could not compare these factors. Also, the prevalence of diabetes and of other medically important conditions was probably under-recorded, and power to analyse these subgroups was limited. In addition, we did not record smoking, which may be a risk factor for surgical site infection. ${ }^{11}$

The diagnosis of infection, although done using guidelines, is subjective and has previously been shown to have inter-observer and intra-observer variation..$^{12}$ The definition we used is the most widely implemented standard definition of wound infection. ${ }^{12}$ Although we asked for recording of patients' compliance at the end of the trial, no recorded instances of non-compliance occurred, which may have been because of inadequate reporting; lack of compliance could also be subjective. We had no reported incidences of patients refusing their allocated intervention, but this could have been because of incomplete reporting rather than perfect compliance. Patients in the intervention group were asked to "bathe as normal" after removal of the dressing, but they did not record the number of episodes of wetting.

\section{Definition of surgical site infection}

- Infection must be within 30 days of excision

- There must be purulent discharge from the wound, or

- The general practitioner must diagnose a wound infection, or

- The general practitioner starts antibiotics

- Stitch abscess must not be counted as an infection
Table 2 Baseline comparison of intervention (wet) and control (dry) group. Values are numbers (percentages) unless stated otherwise

\begin{tabular}{lccc} 
& $\begin{array}{c}\text { Wet (intervention) } \\
(\mathrm{n}=450)\end{array}$ & Dry (control) (n=420) & P value \\
\hline Mean (SD) age (years) & $55.9(16.6)$ & $56.5(16.2)$ & 0.58 \\
\hline Male patients & $249(55)$ & $208(50)$ & 0.08 \\
\hline $\begin{array}{l}\text { Mean (SD) days to removal } \\
\text { of sutures }\end{array}$ & $8.6(2.2)$ & $8.6(2.2)$ & 1 \\
\hline $\begin{array}{l}\text { Presence of diabetes } \\
\begin{array}{l}\text { History of other medical } \\
\text { condition }\end{array}\end{array}$ & $9(2)$ & $14(3)$ & 0.2 \\
\hline $\begin{array}{l}\text { Treated with 1\% lignocaine } \\
\text { adrenaline }\end{array}$ & $8(2)$ & $10(2)$ & 0.5 \\
\hline Excision of skin cancer & $294(65)$ & $411(98)$ & 0.3 \\
\hline Excision at lower limb & $112(25)$ & $289(69)$ & 0.3 \\
\hline
\end{tabular}

*Chronic obstructive pulmonary disease $(\mathrm{n}=8)$, anaemia (1), "aspirin" (2), "steroids" (3),

"warfarin" (2), ischaemic heart disease (1), and peripheral vascular disease (1).

Although we were mainly interested in the wetting of sutures in the immediate postoperative period, separating this factor from uncovering and removing the dressing was difficult in practice, so we measured the two factors together.

Some limits to generalising these findings exist. The general practitioners involved were younger and more predominantly female than the average Australian general practitioner. ${ }^{13}$ The population of Mackay is slightly older and has a lower median household income than the Australian population as a whole. ${ }^{14}$ Mackay is a provincial town in tropical North Queensland. The climate is hot and humid, with the mean daily maximum temperature ranging between $24.2^{\circ} \mathrm{C}$ and $30^{\circ} \mathrm{C}$ during the summer months, and a relative humidity of $75-79 \%{ }^{15}$ These tropical conditions may increase sweat production and produce damp dressings, which might reduce the effectiveness of wound dressings as a potential barrier against exogenous bacteria. ${ }^{16-18}$ This would make the dry and covered control group more prone to infection in a tropical environment. Our findings may therefore not be generalisable to a temperate climate where dressings are less likely to become damp. Our results are encouraging, however, and studies in temperate climate should be considered.

Contributors: $\mathrm{CH}$ conceived and designed the study and analysed and interpreted the data. PB did the sample size calculation and statistical analysis. BR, SB, RB, MC, RS, and DG contributed to the design of the study. All authors contributed to the manuscript. $\mathrm{CH}$ is the guarantor.

Funding: Research was funded by a novice research scholarship from the primary health care research and development fund. The authors' work is independent of this funding.

Competing interests: None declared.

Ethical approval: Approval number H1902 granted by James Cook University ethics committee.

Mangram AJ, Horan TC, Pearson ML, Silver LC, Jarvis WR. Guideline for the prevention of surgical site infection. Infect Control Hosp Epidemiol 1999;20:250-78.

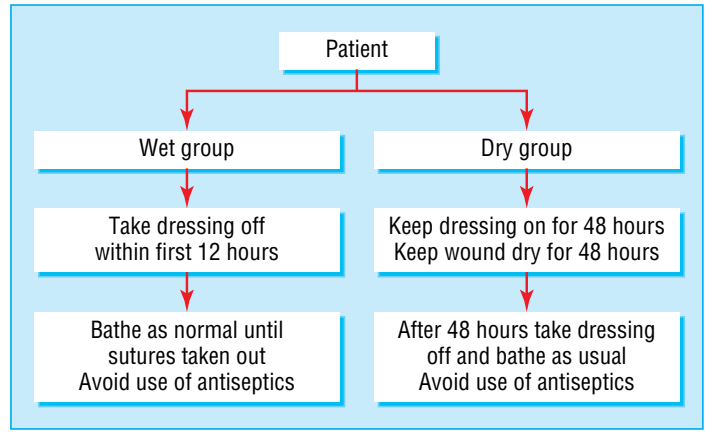

Fig 2 Flowchart of enrolment, randomisation, and follow-up of patients 


\section{Research}

2 Goldberg HM, Rosenthal SA, Nemetz JC. Effect of washing closed head and neck wounds on wound healing and infection. Am J Surg 1981;141:358-9.

3 Noe JM, Keller M. Can sutures get wet? Plast Reconstr Surg 1988;81:82-4.

Carragee EJ, Vittum DW. Wound care after posterior spinal surgery: does early bathing affect the rate of wound complications? Spine 1996;21:2160-2.

5 Neues C, Haas E. Modification of postoperative wound healing by showering. Chirurg 2000;71:234-6.

6 Ajoa OG. Surgical wound infection: a comparison between dressed and undressed wounds.J Trop Med Hyg 1077;80:192-6.

7 Weiss Y. Simplified management of operative wounds by early exposure. Int Surg 1983;68:237-40

8 Merei JM. Pediatric clean surgical wounds: is dressing necessary? J Pediatr Surg 2004:39:1871-3.

9 Lathlean S. Skin cancer in general practice in South Australia. Aust Fam Physician 1999;28(supp 1).

10 Cruse JE, Foord R. The epidemiology of wound infection. Surg Clin North Am $1980 ; 60: 27-39$

\section{What is already known on this topic}

Guidelines instruct that surgical wounds closed primarily should be kept dry and covered for 48 hours

Sparse evidence suggests that wetting and uncovering sutures makes no difference to infection rates

\section{What this study adds}

Wetting and uncovering sutures in the first 48 hours after minor excisions did not increase the infection rate
11 Nagachinta T, Stephens M, Reitz B, Polk BF. Risk factors for surgical wound infection following cardiac surgery. J Infect Dis 1987;156:967-73.

12 Bruce J, Russell EM, Mollison J, Krukowski ZH. The quality of measurement of surgical wound infection as the basis for monitoring: a systematic review. J Hosp Infect 2001;49:99-108

13 Britt H, Miller GC, Knox S, Charles J, Pan Y, Henderson J, et al 2005. General practice activity in Australia 2004-05. Canberra: Australian Institute of Health and Welfare, 2005. (General Practice Series No. 18)

14 CLIB 2001. Basic community profile. 2001 census of population and housing. Australian Bureau of Statistics, 2001.

15 Australian Government, Bureau of Meteorology. Climate averages for Mackay MO. www.bom.gov.au (accessed 5 Sept 2005).

16 Edlich RF. Biochemical and clinical aspects. In: Cohen IK, Diegelman RF, Lindblad WJ, eds. Wound healing. Sydney: WB Saunders, 1992:581-600.

17 Thomas S. Wound management and dressings. London: Pharmaceutical Press, 1990:9-19. 18 Colebrook L, Hood AM. Infection through soaked dressings. Lancet 1948;ii:682-3.

$$
\text { (Accepted } 9 \text { March 2006) }
$$

doi 10.1136/bmj.38800.628704.AE

James Cook University, Queensland, Australia

Clare Heal senior lecturer

Petra Buettner senior lecturer

Beverly Raasch associate professor

Mackay, Queensland, Australia

Sheldon Browning general practitioner

David Graham general practitioner

Rachel Bidgood general practitioner

Margaret Campbell general practitioner

Robert Cruikshank general practitioner

Correspondence to: C Heal, 10 Sunset Beach Court, Shoal Point, Mackay, Old

4750, Australia clarshel@hotmail.com 